\title{
Assessment of the myocardial twisting motion by through-plane velocity-mapping in long axis geometry
}

\author{
Anja Lutz*1, Axel Bornstedt ${ }^{1}$, Peter Bernhardt ${ }^{1}$, Vinzenz Hombach ${ }^{1}$, \\ Nico Merkle ${ }^{1}$, Robert Manzke ${ }^{2}$ and Volker Rasche ${ }^{1}$
}

\begin{abstract}
Address: ${ }^{1}$ University Hospital of Ulm, Ulm, Germany and ${ }^{2}$ Philips Research Europe, Hamburg, Germany
\end{abstract}
* Corresponding author

from 13th Annual SCMR Scientific Sessions

Phoenix, AZ, USA. 21-24 January 2010

Published: 21 January 2010

Journal of Cardiovascular Magnetic Resonance 20 I0, I 2(SuppI I):P83 doi:I0.I 186/1532-429X-12-SI-P83

This abstract is available from: http://jcmr-online.com/content/I2/SI/P83

(c) 2010 Lutz et al; licensee BioMed Central Ltd.

\section{Introduction}

Several studies have revealed the relevance of assessing the rotational motion of the heart, especially for selection of DCM patient for CRT therapy, in which a reduced LV twisting was found to be predictive for the response [1,2].

\section{Purpose}

The purpose was to evaluate the possibility of combining conventional CINE imaging techniques in long axes geometry with through-plane velocity-encoding for fast simultaneous assessment of the twisting motion.

\section{Methods}

The protocol was performed in 3 healthy volunteers. All imaging was performed on a $3 \mathrm{~T}$ whole-body-scanner (Achieva, Philips Medical Systems, Netherlands) with a segmented gradient echo phase contrast black blood imaging sequence. 6 long axis views were acquired at $30^{\circ}$ angular spacing. The imaging parameters were: $\mathrm{TR} / \mathrm{TE}=$ $6.8 \mathrm{~ms} / 4.6 \mathrm{~ms}$, resolution $2 \times 2 \times 8 \mathrm{~mm}^{3}$, flip angle $15^{\circ}$, VENC $=15 \mathrm{~cm} / \mathrm{s}$ in through plane direction, 30 heart phases. Black-blood contrast was achieved by the application of two alternating saturation-prepulses with $5 \mathrm{~mm}$ distance to the imaged slice. Image acquisition time for each slice was 1:04 minutes. Quantitative through-plane motion analysis was performed by an in-house developed analysis software. After manual segmentation of the left ventricle the rotation angle $\phi$ was calculated for apical, equatorial and basal segments and compared for retrieval of the twisting motion component.

\section{Results}

Figure 1 shows the anatomical (a) and c)) and velocity encoded (b) and d)) images exemplarily for one of the 6 long axis views of one volunteer. The opposing rotational direction of the apex and the basis is clearly revealed in both images. Figure 1e) displays the resulting rotation curves for the myocardial lateral wall. The twist angle (curve blue) shows the typical systolic twisting and diastolic untwisting. The twist angle was the minimal in the septal wall as also described in [3].

\section{Discussion}

It has been shown [4] that CINE imaging of 6 long axes (LAX) can be applied for retrieving the regional wall motion score. In this contribution it was shown that the LAX technique can be combined with through-plane motion-encoding, which enables additional assessment of the twisting motion of the myocardium. The combination of both techniques appears promising for the simultaneous identification of the contraction impairments and its impact on the twisting motion, which might enable improved identification of patients prone to respond to CRT.

\section{References}

I. Rüssel, et al.: JACC: Cardiovascular Imaging 2009, 2(5):648-655.

2. Sade, et al: Am J Cardiol 2008, I0 1: I I63-9.

3. Young, et al.: Circulation 1994, 90(3): 1584.

4. Bernhardt, et al:: in press. 

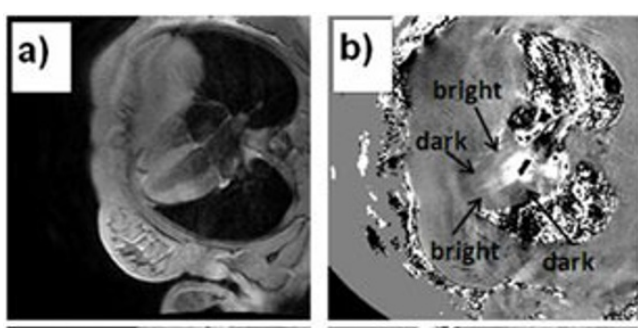

e)
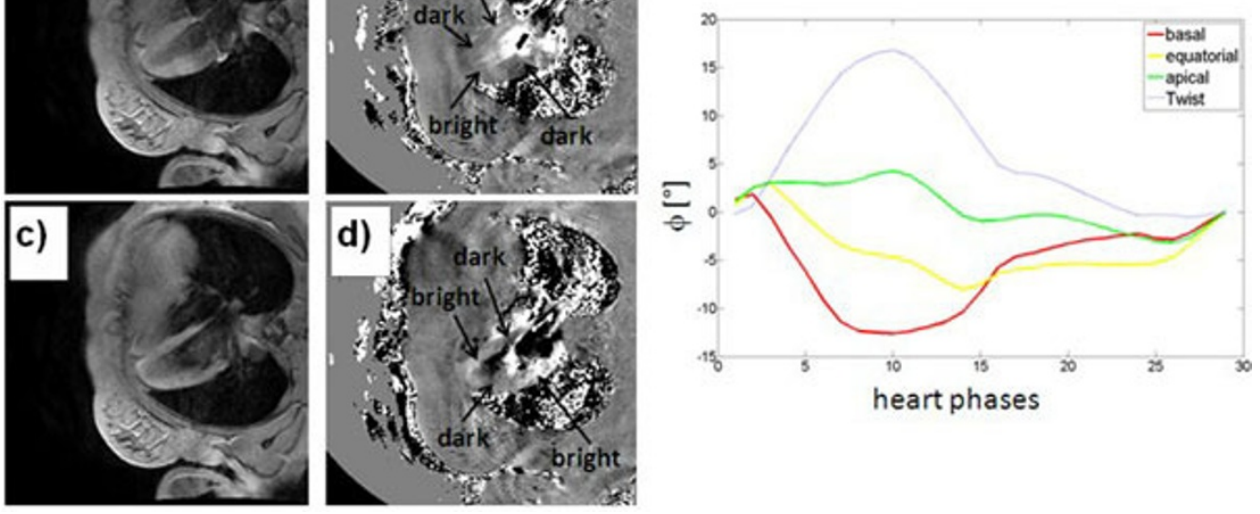

Figure I

a) anatomical and b) velocity image at endsystole (heart phase II); c) anatomical and d) velocity image at diastole (heart phase 6). In both velocity images the velocity twist is visible. In b) the velocity direction of the apical heart is counterclockwise in respect to the short axes geometry and the basal velocity is vice versa. In d) the velocity direction is changed. le) displays the angle of the myocardial free wall, by which it is rotated. Red: basal rotation; green: apical rotation; yellow: equatorial rotation and blue: twist angle $=$ phi $_{\text {apical }}-$ phi $_{\text {basal }}$. 